\title{
Proton Beam Therapy for Local Recurrence of Rectal Cancer
}

\author{
YUICHI HIROSHIMA ${ }^{1}$, HITOSHI ISHIKAWA ${ }^{1,2}$, MOTOHIRO MURAKAMI ${ }^{1}$, \\ MASATOSHI NAKAMURA ${ }^{1}$, SHOSEI SHIMIZU ${ }^{1}$, TSUYOSHI ENOMOTO ${ }^{3}$, TATSUYA ODA ${ }^{3}$, \\ MASASHI MIZUMOTO ${ }^{1}$, KEI NAKAI $^{1}$, TOSHIYUKI OKUMURA ${ }^{1}$ and HIDEYUKI SAKURAI ${ }^{1}$ \\ ${ }^{1}$ Department of Radiation Oncology, Proton Medical Research Center, University of Tsukuba Hospital, Tsukuba, Japan; \\ ${ }^{2}$ QST hospital, National Institutes for Quantum and Radiological Science and Technology, Chiba, Japan; \\ ${ }^{3}$ Department of GI \& HBP Surgery, University of Tsukuba Hospital, Tsukuba, Japan
}

\begin{abstract}
Background/Aim: Curing local recurrence of rectal cancer (LRRC) is difficult with conventional photon radiotherapy. Proton beam therapy (PBT) on the other hand, has unique physical characteristics that permit higher doses to LRRC while minimizing side effects on surrounding organs. However, the efficacy of PBT on controlling rectal cancer recurrence has not yet been reported. This study aimed to evaluate clinical outcomes and toxicities of PBT for LRRC. Patients and Methods: Clinical outcomes were retrospectively evaluated for 12 patients with 13 total lesions that had received PBT for LRRC at our institute. Results: The median follow-up period from the initiation of PBT was 42.9 months. The 3-year local control, progression-free survival and overall survival rates were $80.2 \%, 12.1 \%$ and $71.3 \%$, respectively. Median survival time was 67.1 months. There were no severe acute or late adverse events. Conclusion: PBT could be a safe and effective treatment method for LRRC.
\end{abstract}

In Japan, colorectal cancer was the most common malignancy in 2017 and the second-leading cause of death in 2018 (1). Rectal cancer accounted for about one-third of these, with 51,238 patients in 2017 and 15,244 deaths in 2018 (1). Advances in surgical techniques have decreased the frequency of postoperative local recurrence (LR); however, LR persists in 5$11 \%$ of patients even after curative resection of rectal cancer (2). Surgery, especially complete surgical resection (R0 resection), is

This article is freely accessible online.

Correspondence to: Yuichi Hiroshima, Department of Radiation Oncology \& Proton Medical Research Center, Faculty of Medicine, University of Tsukuba, 2-1-1, Amakubo, Tsukuba 3058576 , Japan. Tel: +81 298537100, Fax: +81 298537102, e-mail: hiroshima@pmrc.tsukuba.ac.jp

Key Words: Rectal cancer, local recurrence, proton beam therapy, dose escalation, chemoradiotherapy, particle beam therapy. the only curative treatment for LR of rectal cancer (LRRC) but there are severe complications during and after resection, meaning that few patients with LRRC can undergo R0 resection (3-5). In Japan, surgery for resection is recommended for LRRC only when $\mathrm{R} 0$ resection is possible (6).

Some LRRC patients who do not qualify for surgical resection therefore receive radiotherapy, especially for symptoms like bleeding and pain. However, conventional photon radiotherapy at 50.0 Gray (Gy) and 25 fractions is palliative, not curative, as LRRC has very low radiosensitivity (7). In these cases, dose escalation may improve clinical outcomes but close proximity of these tumors to radiosensitive organs like the colon, bladder and small intestine prevent this approach for conventional photon radiotherapy (8).

Proton beam therapy (PBT), on the other hand, has unique physical characteristics, such as the Bragg-Peak, in which the proton beam creates a localized peak in a deep part of the body according to the input energy. Because of this feature, PBT can provide higher doses to LRRC, while minimizing unnecessary irradiation of the surrounding, radiosensitive organs. In recent decades, the efficacy and feasibility of PBT has been reported for diverse cancers such as the liver, esophagus, or pancreas (9-15).

We treated 12 patients with curative PBT in our institute and we report here the results demonstrating the potential effectiveness of PBT for patients with LRRC.

\section{Patients and Methods}

Patients. PBT for LRRC was conducted for 12 patients with 13 total lesions between 2003 and 2018 at our institute. Before beginning treatment, LRRC without distant metastasis was verified by computed tomography (CT), magnetic resonance imaging (MRI) and F-18-2-Fluoro-2-deoxy-D- glucose (FDG) positron emission tomography (PET). After curative resection of the primary tumor and regional lymph nodes, rectal adenocarcinoma without gross or microscopic residual disease was confirmed. After the examinations were completed, all cases in which complete resection was difficult were evaluated by surgeons on the cancer board. 


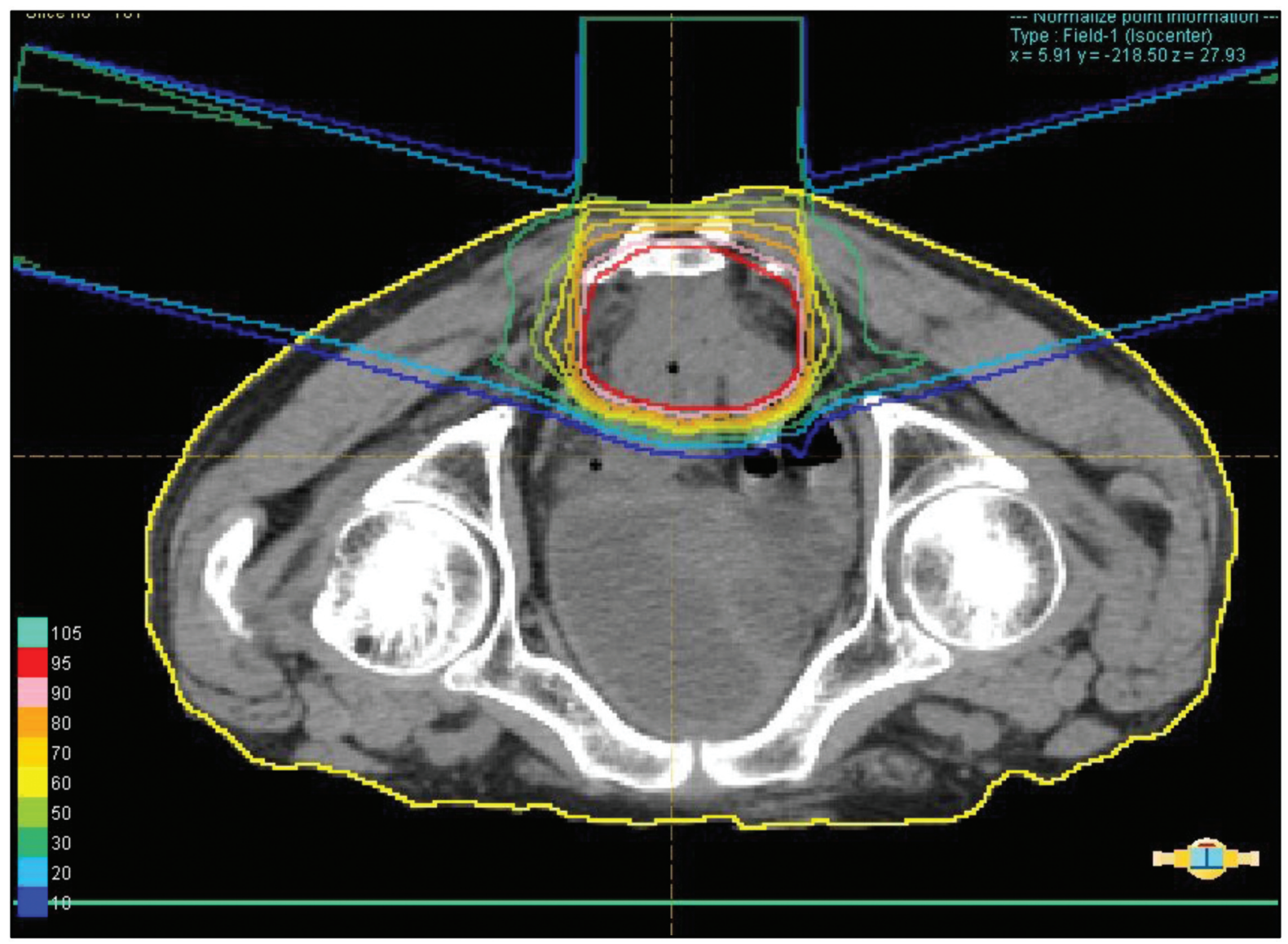

Figure 1. Dose distribution of proton beam therapy for sacral anterior LRRC at a total dose of 75 GyE in 25 fractions. The supine position resulted in the gantry being closer to the lesion and further improved the dose distribution.

As exclusion criteria, patients with an ECOG Performance Status (PS) $>2$, who had received prior photon or particle radiation therapy to the same site, who had another primary malignancy, or who had an infection at the tumor site were not included in this study.

Proton beam therapy. Before making a treatment plan, CT images without intravenous contrast agent were taken at $2.5 \mathrm{~mm}$ intervals for planning. We defined the gross tumor volume as the primary tumor while the clinical target volume (CTV) was judged to be an additional $5 \mathrm{~mm}$ margin around the gross tumor volume. Beamdependent margins, such as a $0.5-1 \mathrm{~cm}$ margin around the CTV, were directly added.

Irradiation was performed daily during weekdays and at least 4 days a week, even on holidays. During treatment, all patients were treated with $155-230 \mathrm{MeV}$ proton beams, using a passive spreading method, shaped with ridge filters, double scattering sheets, multicollimators and a custom-made bolus to ensure that the beams conformed to the planning data. Spinal bones and 2 sets of orthogonal digital radiographs were used for daily positional confirmation.

We selected beam fractionations by tumor location, distance between tumors and organs at risk. When the tumors were in the vicinity of high-risk organs, fractional doses were reduced to avoid severe adverse effects. Irradiated doses were calculated by assuming the RBE to be 1.1. Figure 1 depicts a representative case of a LRRC patient and dose distribution of PBT at a total dose of $75 \mathrm{GyE}$ in 25 fractions.

Concurrent chemotherapy. Six of 13 patients in this analysis received concurrent S-1 (combination of Tegafur, Gimeracil and Oteracil). Oral S-1 was prescribed twice daily, but only on the day of irradiation, according to body-surface area $\left(<1.25 \mathrm{~m}^{2}, 80 \mathrm{mg}\right.$ /day; $>1.25$ to $<1.5 \mathrm{~m}^{2}, 100 \mathrm{mg} /$ day; $>1.5 \mathrm{~m}^{2}, 120 \mathrm{mg} /$ day), and was skipped when the absolute granulocyte count was less than $2,000 / \mathrm{mm}^{3}$ or the platelet count was less than $70,000 / \mathrm{mm}^{3}$ on a scheduled dosage day, in cases of poor performance status, abnormal biochemical data, or in the presence of any diagnosed condition that contraindicated administration.

Others were treated solely by PBT if S-1 or fluorouracil was ineffective in a previous treatment cycle or the gastrointestinal tract was in close proximity.

Follow-up procedure. During PBT treatment, acute treatment-related toxicities and treatment efficacies were evaluated once or twice per week in all patients. All patients were scheduled for examination 
Table I. Characteristics of patients and tumors.

\begin{tabular}{lcc}
\hline & Number & $\%$ \\
\hline Patient/lesion number & $12 / 13$ & \\
Median age (range) & $69(38-77)$ & \\
Gender & & \\
$\quad$ Male & 12 & 100.0 \\
Female & 0 & 0 \\
PS & 5 & 41.7 \\
0 & 7 & 58.3 \\
$>1$ & & \\
Surgery & 6 & 50.0 \\
APR & 6 & 50.0 \\
LAR & & \\
Pathologic subtype & 8 & 66.7 \\
Adenocarcinoma & 1 & 8.3 \\
Others & 3 & 25.0 \\
Unknown & & \\
Primary tumor stage (UICC 7th) & 4 & 83.3 \\
IIA & 1 & 8.3 \\
IIIA & 4 & 16.7 \\
IIIB & 1 & 84.6 \\
IIIIC & 2 & 15.4 \\
IVB & & \\
Site of LR & 11 & \\
Presacral & 2 & \\
Side wall of pelvis & 63 mm (50-79.2 mm) \\
Median tumor size (range) & & \\
\hline
\end{tabular}

PS: Eastern Cooperative Oncology Group (ECOG) performance status; APR: abdominal perineal resection; LAR: low anterior resection; LR: local recurrence.

every 3 months and physical condition, blood sampling and imaging were conducted prior to their visits if their physical condition was good after completing PBT. If the patients were unable to visit our institute due to the traveling distance or a poor general condition, follow up of imaging and blood tests was performed at a nearby institute and the results were sent to our hospital. Adjuvant therapy after PBT was done at the discretion of the attending physician in accordance with individual physical condition.

Evaluation and stastical analysis. Acute and late toxicities were evaluated according to the National Cancer Institute Common Terminology Criteria for Adverse Events (CTCAE, version 4.0) (16) while relapse was evaluated according to the Response Evaluation Criteria in Solid Tumors (RECIST, version 1.1) (17). We examined overall survival (OS), progression free survival (PFS), and local control (LC) rates using the Kaplan-Meier method. All analyses were performed using SPSS version 25.0 (IBM Inc. Armonk, NY, USA).

Ethics. All procedures involving human participants, including case reviews of treatments, were conducted in accordance with the ethical standards of the 1964 Declaration of Helsinki and its later amendments and approved by the University of Tsukuba Institutional Research Committee (Approval \#R01-230). All treatments were discussed at an in-hospital conference consisting of surgeons, internists and radiologists; informed consent was obtained from all participants included in the study. We got informed consent from
Table II. Characteristics of treatment plans.

\begin{tabular}{lcccc}
\hline & $\begin{array}{c}\text { Total dose } \\
\text { (GyE) }\end{array}$ & Fractionation & $\begin{array}{c}\text { BED for a/b } \\
\text { ratio } 10\end{array}$ & $\begin{array}{c}\text { Concurrent } \\
\text { chemotherapy }\end{array}$ \\
\hline$\# 1$ & 50.0 & 20 & 62.5 & No \\
$\# 2$ & 50.0 & 20 & 62.5 & No \\
$\# 3$ & 60.0 & 30 & 72 & No \\
$\# 4$ & 60.0 & 20 & 78 & Yes \\
$\# 5$ & 70.0 & 35 & 84 & No \\
$\# 6$ & 76.0 & 38 & 91.2 & No \\
$\# 7$ & 72.0 & 24 & 93.6 & No \\
$\# 8$ & 72.6 & 22 & 96.6 & Yes \\
$\# 9$ & 75.0 & 25 & 97.5 & Yes \\
$\# 10$ & 75.0 & 25 & 97.5 & No \\
$\# 11$ & 72.0 & 18 & 100.8 & Yes \\
$\# 12$ & 72.0 & 18 & 100.8 & Yes \\
$\# 13$ & 79.2 & 24 & 105.3 & Yes \\
\hline
\end{tabular}

GyE: Gray equivalent; BED: biologically effective dose.

either living patients themselves or legally designated next-of-kin where appropriate.

\section{Results}

Patient characteristics. From 2001 to 2018, 12 patients with 13 total LRRC lesions were included. Six of 13 patients in this analysis were treated by curative chemotherapy concurrent with PBT while the remaining 7 received only PBT and all patients completed their scheduled treatments. Patient characteristics are summarized in Table I. The median age was 69 years (range $=38-77$ years) and all patients were male. Relapse locations included the presacral region $(n=12)$ and pelvic side walls $(n=1)$. The median tumor size was $63 \mathrm{~mm}$ (range $=20-131 \mathrm{~mm}$ ). The median time from surgery to LR was 23.5 months (range=2.1-89.3 months). Total irradiated doses and fractionations are summarized in Table II.

Treatment outcomes. The median follow-up period from the initiation of PBT was 42.9 months (range=3.6-102.6 months). As of November 2020, a total of 6 patients had died of rectal cancer. Recurrence after PBT was observed in 10 patients $(83.3 \%)$, of whom $2(16.7 \%)$ had a LR, 7 $(58.3 \%)$ had distant metastasis and $1(8.3 \%)$ had both. The 3-year LC, PFS and OS rates were $80.2 \%$ [95\% confidence interval $(\mathrm{CI})=55.1-100 \%], 12.1 \%(95 \% \mathrm{CI}=0-33.7 \%)$ and $71.3 \%(95 \% \mathrm{CI}=43.7-98.9 \%)$, respectively. Median survival time was 67.1 months $(95 \%$ CI=24.0-110.2 months) (Figure 2).

Toxicities. Treatment-related toxicities are described in Table III. No grade $>2$ acute or late adverse events were observed over the follow-up time period. 

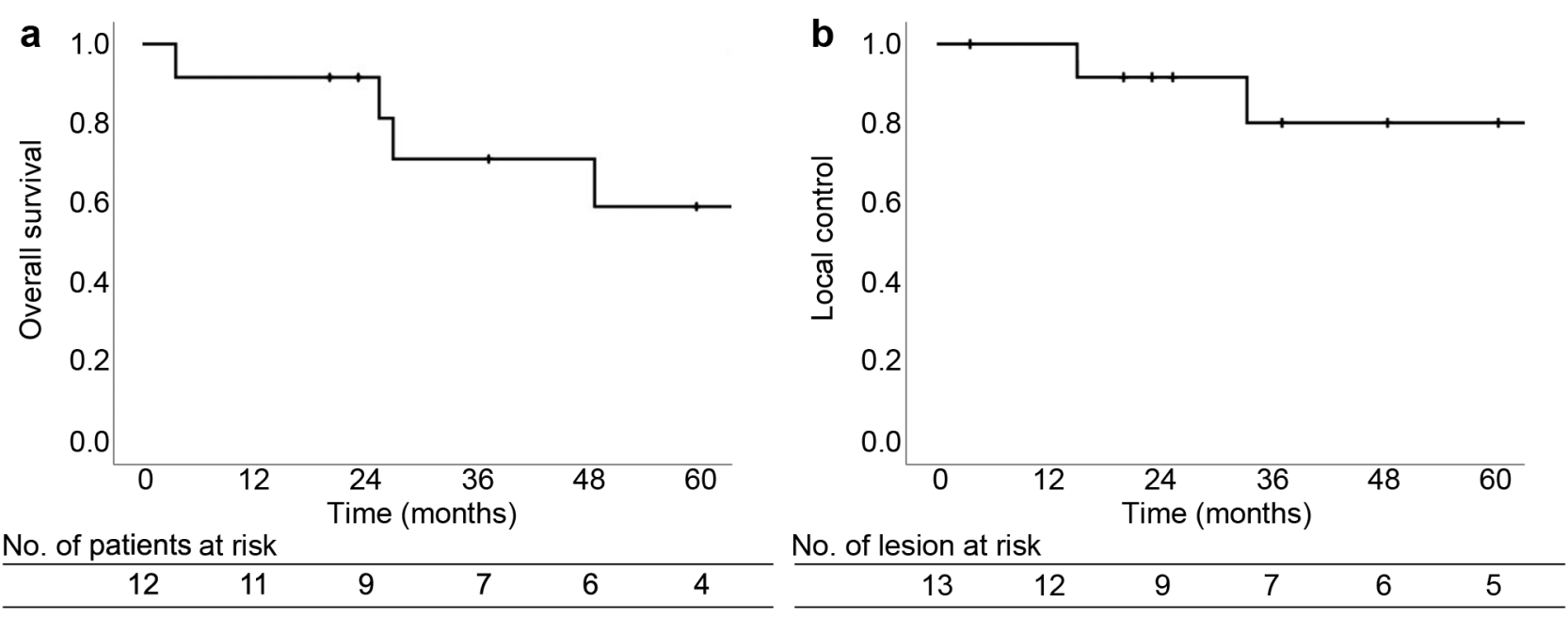

Figure 2. Kaplan-Meier estimates of (a) overall survival (OS) and (b) local control (LC) rates from the initiation of proton beam therapy.

\section{Discussion}

In our study, the 3-year OS and LC rates were $71.3 \%$ and $80.2 \%$. Although from a small population, these results were encouraging since the enrolled patients were not suitable for radical resection.

Westberg et al. showed some factors that influence death risk after curative treatment for LRRC from a large population-based national patient cohort with long follow up (18). They reported that patients aged 80 years and over, with stage III primary tumors, symptoms, hydronephrosis, or a non-central LRRC were at a significant high risk with regards to curative surgery. In our study, 8 of 12 patients were at a primary tumor stage of more than III and this fact suggests that particle beam therapy may be safe and effective in some of these cases.

It could be hard to achieve R0 resection in LRRC that have infiltrated the pelvic side or posterior wall as centrally located LRRC, because they tend to invade organs at risk such as the gastrointestinal tract and bladder. As a result, centrally located LRRC with a good indication for surgery tends to not be recommended for PBT in order to limit dose escalation around organs at risk. On the other hand, since a non-centrally located LRRC tends to be distant from organs at risk, this may be a good indication for PBT when taking into account the risks of mortality and morbidity following curative surgery. In our study, although 11 of 13 lesions were located centrally, there were no serious acute or late adverse events. We tried to administer treatment plans that had been redesigned several times to deliver the highest possible dose to the LRRC while suppressing exposure to at-risk organs, thereby helping to alleviate severe adverse events.
Table III. Treatment-related acute and late adverse events.

\begin{tabular}{|c|c|c|c|c|c|c|c|c|c|}
\hline \multirow[b]{2}{*}{ Toxicity } & \multirow[b]{2}{*}{$\mathrm{n}$} & \multicolumn{4}{|c|}{ Acute } & \multicolumn{4}{|c|}{ Late } \\
\hline & & Gr0 & Gr1 & Gr2 & Gr3 & Gr0 & Gr1 & Gr2 & Gr3 \\
\hline Skin & 13 & 4 & 9 & 0 & 0 & 13 & 2 & 0 & 0 \\
\hline GI tract & 13 & 13 & 0 & 0 & 0 & 13 & 0 & 0 & 0 \\
\hline Urinary & 13 & 13 & 0 & 0 & 0 & 13 & 0 & 0 & 0 \\
\hline
\end{tabular}

All adverse events were evaluated by CTCAE 4.0.

Photon radiotherapy is sometimes suggested for patients who are not suitable for surgical resection, but radiosensitive organs around the tumor make sufficient curative doses difficult even with high-precision radiotherapy techniques such as intensity modulated radiotherapy (IMRT). Tanaka $e t$ al. reported that 3-year OS and LC rates with 3-dimensional conformal radiotherapy were $45.2 \%$ and $19.6 \%$ and found that doses higher than the biological effective dose (BED) of $75 \mathrm{~Gy}$ brought better clinical outcomes (7). However, this exceeds the tolerable dose for risk organs, such as the gastrointestinal tract, and would increase the frequency of serious adverse events like perforation. Although achieving therapeutically sufficient doses is sometimes difficult for photon radiotherapy, particle beam therapy may more easily achieve clinical goals due to the Bragg Peak effect. In fact, no serious adverse effects were observed in all 10 patients given at $>$ BED of 75 Gy in our study.

Proton beam therapy for LRRC has been reported in a small number of cases; Lee et al. performed PBT on 4 of 67 LRRC patients (19). Although there are no reports on the 
results of PBT, they noted that they were able to prescribe high doses for patients with very close to organs at risk, such as small bowel. Hamauchi et al. reported the results of PBT in 13 LRRC patients with $70 \mathrm{GyE}$ (20). With median LC and PFS of 504 and 404 days, respectively, and only one patient with a Grade 3 adverse event, they suggested that PBT may be a treatment option for LRRC due to good local control and low incidence of serious adverse events. Berman et al. reported the outcomes of PBT in 7 LRRC patients with prior irradiation and the dose distribution compared to IMRT (21). The average prescribed dose of PBT was $61.2 \mathrm{GyE}$, with complete response and progressive disease in 1 patient each and partial response in 7 patients. In terms of dose distribution, PBT was significantly better than IMRT, especially in avoiding the small intestine. All these reports with PBT are consistent with the present report, as they safely delivered high doses to the LRRC while lowering the dose delivered to the gastrointestinal tract.

There are some reports of carbon-ion radiation therapy (CIRT) for LRRC. CIRT is a particle beam therapy and is characterized by high BED (22). Yamada et al. reported a phase $1 / 2$ dose escalation study (23) where the 3 -year and 5year OS rates were $72 \%$ and $53 \%$. In phase I, they demonstrated that 73.6 Gy (RBE: Relative Biological Effect weighted absorbed dose) in 16 fractions was better and carried this over to a phase 2 study. Shinoto et al. reported a multi-institutional study of CIRT on LRRC in which three CIRT institutions in Japan were included (24). From a total number of 224 patients, they reported 3-year and 5-year OS of rates $73 \%$ and $51 \%$. They also reported that grade 3 acute toxicities were observed in $3(1 \%)$ of the 224 patients (1 gastrointestinal reaction and 2 pelvic abscesses) while grade 3 late toxicities were observed in 12 patients (5\%) (2 skin reactions, 2 gastrointestinal reactions, 1 peripheral nerve reaction and 7 pelvic abscesses). Most patients received 73.6 Gy (RBE) in 16 fractions and, as their results were very similar to the study by Yamada et al., these reports point to better clinical outcomes and lower toxicity than conventional photon radiotherapy.

With regard to biological effects, PBT is similar to photon beam therapy (25) in that more dose escalation may result in better clinical outcomes, as seen in CIRT studies; however, even PBT may face positioning difficulties between tumor and gastrointestinal tract locations. To improve clinical outcomes, local hyperthermia is an option as it is known to heat tumors, increasing the blood supply and improving delivery of large doses of oxygen and drugs to tumors and enhancing the effects of radiotherapy $(26,27)$. It also has a function as a radiation- and chemo-sensitizer while inhibiting transcription factors (28). Because LRRC is characterized by chemo-and radio- resistance and hypoxia, these patients are presumably well adapted for combination of hyperthermic therapy with lower linear energy transfer radiation such as
PBT (29). Thus, we have recently started concomitant use of hyperthermia in PBT for LRRC to improve outcomes. Due to the short accumulation period and the small number of patients, reports from our institute on this promising effect remain forthcoming.

Another solution to improve clinical outcomes is a surgical spacer. The method of using a tissue expander for the spacer has been reported for a long time, but these need to be removed after irradiation. Kemmochi et al. reported on experiences of surgical spacer placement using polytetrafluoroethylene and polyurethane (30), pointing out that artificial objects adhere to the digestive tract and damage it due to friction after a long insertion period. Akasaka et al. reported on the efficacy and safety of making distance between the tumor and the at-risk organ by using a nonwoven spacer produced with a biocompatible, synthetic polymeric material called polyglycolic acid (PGA) (31). The characteristic of these spacers is that the size does not change for 8 weeks and is gradually absorbed from about 12 weeks with no toxicity, including no C-reactive protein (CRP) elevation and no liver or renal dysfunction. Since 2019, this spacer can be used for particle beam therapy within the national insurance system of Japan and it is expected that spacer-enabled higher doses can be administered, thereby increasing the number of treatable LRRC patients.

There are some limitations to this study, including the retrospective design, small patient population and possible selection bias. Additionally, we did not compare PBT with photon radiotherapy and CIRT directly; therefore, it is unclear how much better PBT actually is. However, we found good local control and overall survival without severe adverse effects by using PBT for LRRC. These results suggest that PBT could be an effective and safe definitive therapy for LRRC.

\section{Conclusion}

Our result showed that PBT for LRRC resulted in longer survival and better local control than previously reported photon radiotherapy without any severe adverse events. Therefore, PBT could be a safe and effective treatment method for LRRC.

\section{Conflicts of Interest}

The Authors declare no conflicts of interest.

\section{Authors' Contributions}

Conceptualization, Y.H. and H.S.; methodology, Y.H.; formal analysis, M.M. (Motohiro Murakami); investigation, Y.H. and H.I.; resources, M.N.; data curation, S.S.; writing-original draft preparation, Y.H.; writing - review and editing, H.I. and M.M. (Masashi Mizumoto); visualization, K.N.; supervision, T.O. 
(Toshiyuki Okumura) and T.E.; project administration, H.S. and T.O. (Tatsuya Oda). All authors have read and agreed to the published version of the manuscript.

\section{Acknowledgements}

The Authors would like to thank Dr. Bryan J. Mathis of the University of Tsukuba Medical English Communications Center for language revision. This work was partially supported by Grants-inAid for Scientific Research (B) (19H03596) from the Ministry of Education, Culture, Sports, Science and Technology of Japan.

\section{References}

1 Cancer registry and statistics. Cancer information service, national cancer center, Japan (ministry of health, labour and welfare, national cancer registry), 2020. Available at: https:// ganjoho.jp/reg_stat/statistics/dl/index.html [Last accessed on May 26, 2021]

2 van Gijn W, Marijnen CA, Nagtegaal ID, Kranenbarg EM, Putter H, Wiggers T, Rutten HJ, Påhlman L, Glimelius B, van de Velde CJ and Dutch Colorectal Cancer Group: Preoperative radiotherapy combined with total mesorectal excision for resectable rectal cancer: 12-year follow-up of the multicentre, randomised controlled TME trial. Lancet Oncol 12(6): 575-582, 2011. PMID: 21596621. DOI: 10.1016/S1470-2045(11)70097-3

3 Nielsen MB, Rasmussen PC, Lindegaard JC and Laurberg S: A 10-year experience of total pelvic exenteration for primary advanced and locally recurrent rectal cancer based on a prospective database. Colorectal Dis 14(9): 1076-1083, 2012. PMID: 22107085. DOI: 10.1111/j.1463-1318.2011.02893.x

4 Nielsen M, Rasmussen P, Pedersen B, Hagemann-Madsen R, Lindegaard $\mathrm{J}$ and Laurberg S: Early and late outcomes of surgery for locally recurrent rectal cancer: a prospective 10-year study in the total mesorectal excision era. Ann Surg Oncol 22(8): 26772684, 2015. PMID: 25564165. DOI: 10.1245/s10434-014-4317-y

5 Bhangu A, Ali SM, Darzi A, Brown G and Tekkis P: Metaanalysis of survival based on resection margin status following surgery for recurrent rectal cancer. Colorectal Dis 14(12): 14571466, 2012. PMID: 22356246. DOI: 10.1111/j.1463-1318.2012. 03005.x

6 Hashiguchi Y, Muro K, Saito Y, Ito Y, Ajioka Y, Hamaguchi T, Hasegawa K, Hotta K, Ishida H, Ishiguro M, Ishihara S, Kanemitsu Y, Kinugasa Y, Murofushi K, Nakajima TE, Oka S, Tanaka T, Taniguchi H, Tsuji A, Uehara K, Ueno H, Yamanaka T, Yamazaki K, Yoshida M, Yoshino T, Itabashi M, Sakamaki K, Sano K, Shimada Y, Tanaka S, Uetake H, Yamaguchi S, Yamaguchi N, Kobayashi H, Matsuda K, Kotake K, Sugihara K and Japanese Society for Cancer of the Colon and Rectum: Japanese Society for Cancer of the Colon and Rectum (JSCCR) guidelines 2019 for the treatment of colorectal cancer. Int J Clin Oncol 25(1): 1-42, 2020. PMID: 31203527. DOI: 10.1007/s10147-019-01485-z

7 Tanaka H, Yamaguchi T, Hachiya K, Okada S, Kitahara M, Matsuyama K and Matsuo M: Radiotherapy for locally recurrent rectal cancer treated with surgery alone as the initial treatment. Radiat Oncol J 35(1): 71-77, 2017. PMID: 28395503. DOI: 10.3857/roj.2016.02005

8 Höckel M, Schlenger K, Höckel S, Aral B, Schäffer U and Vaupel $\mathrm{P}$ : Tumor hypoxia in pelvic recurrences of cervical cancer. Int $\mathrm{J}$
Cancer 79(4): 365-369, 1998. PMID: 9699528. DOI: 10.1002/ (sici)1097-0215(19980821)79:4<365::aid-ijc10>3.0.co;2-4

9 Tamura S, Okamura Y, Sugiura T, Ito T, Yamamoto Y, Ashida R, Ohgi K, Murayama S and Uesaka K: A comparison of the outcomes between surgical resection and proton beam therapy for single primary hepatocellular carcinoma. Surg Today 50(4): 369-378, 2020. PMID: 31602531. DOI: 10.1007/s00595-01901888-5

10 Lin SH, Hobbs BP, Verma V, Tidwell RS, Smith GL, Lei X, Corsini EM, Mok I, Wei X, Yao L, Wang X, Komaki RU, Chang JY, Chun SG, Jeter MD, Swisher SG, Ajani JA, Blum-Murphy M, Vaporciyan AA, Mehran RJ, Koong AC, Gandhi SJ, Hofstetter WL, Hong TS, Delaney TF, Liao Z and Mohan R: Randomized phase IIB trial of proton beam therapy versus intensity-modulated radiation therapy for locally advanced esophageal cancer. J Clin Oncol 38(14): 1569-1579, 2020. PMID: 32160096. DOI: 10.1200/JCO.19.02503

11 Anakotta RM, van der Laan HP, Visser S, Ribeiro CO, Dieters M, Langendijk JA, Both S, Korevaar EW, Sijtsema NM, Knopf A and Muijs CT: Weekly robustness evaluation of intensitymodulated proton therapy for oesophageal cancer. Radiother Oncol 151: 66-72, 2020. PMID: 32663538. DOI: 10.1016/ j.radonc.2020.07.015

12 Hiroshima Y, Fukumitsu N, Saito T, Numajiri H, Murofushi KN, Ohnishi K, Nonaka T, Ishikawa H, Okumura T and Sakurai H: Concurrent chemoradiotherapy using proton beams for unresectable locally advanced pancreatic cancer. Radiother Oncol 136: 37-43, 2019. PMID: 31015127. DOI: 10.1016/j.radonc.2019.03.012

13 Igaki H, Mizumoto M, Okumura T, Hasegawa K, Kokudo N and Sakurai H: A systematic review of publications on charged particle therapy for hepatocellular carcinoma. Int J Clin Oncol 23(3): 423-433, 2018. PMID: 28871342. DOI: 10.1007/s10147017-1190-2

14 Ishikawa H, Hashimoto T, Moriwaki T, Hyodo I, Hisakura K, Terashima H, Ohkohchi N, Ohno T, Makishima H, Mizumoto M, Ohnishi K, Okumura T and Sakurai H: Proton beam therapy combined with concurrent chemotherapy for esophageal cancer. Anticancer Res 35(3): 1757-1762, 2015. PMID: 25750339.

15 Terashima K, Demizu Y, Hashimoto N, Jin D, Mima M, Fujii O, Niwa Y, Takatori K, Kitajima N, Sirakawa S, Yonson K, Hishikawa Y, Abe M, Sasaki R, Sugimura K and Murakami M: A phase I/II study of gemcitabine-concurrent proton radiotherapy for locally advanced pancreatic cancer without distant metastasis. Radiother Oncol 103(1): 25-31, 2012. PMID: 22300608. DOI: $10.1016 /$ j.radonc.2011.12.029

16 US Department of Health and Human Services: Common terminology criteria for adverse events (ctcae) version 4.0. National Institutes of Health, National Cancer Institute, 2009. Available at: https://evs.nci.nih.gov/ftp1/CTCAE/CTCAE_4.03/Archive/CTCAE _4.0_2009-05-29_QuickReference_8.5x11.pdf [Last accessed on May 26, 2021]

17 Eisenhauer EA, Therasse P, Bogaerts J, Schwartz LH, Sargent D, Ford R, Dancey J, Arbuck S, Gwyther S, Mooney M, Rubinstein L, Shankar L, Dodd L, Kaplan R, Lacombe D and Verweij J: New response evaluation criteria in solid tumours: revised RECIST guideline (version 1.1). Eur J Cancer 45(2): 228-247, 2009. PMID: 19097774. DOI: 10.1016/j.ejca.2008.10.026

18 Westberg K, Palmer G, Hjern F, Nordenvall C, Johansson H, Holm $\mathrm{T}$ and Martling A: Population-based study of factors 
predicting treatment intention in patients with locally recurrent rectal cancer. Br J Surg 104(13): 1866-1873, 2017. PMID: 29023631. DOI: 10.1002/bjs.10645

19 Lee JH, Kim DY, Kim SY, Park JW, Choi HS, Oh JH, Chang HJ, Kim TH and Park SW: Clinical outcomes of chemoradiotherapy for locally recurrent rectal cancer. Radiat Oncol 6: 51, 2011. PMID: 21595980. DOI: 10.1186/1748-717X-6-51

20 Hamauchi S, Yamazaki K, Yasui H, Boku N, Onozawa Y, Fukutomi A, Machida N, Yokota T, Todaka A, Taniguchi H, Tsushima T, Funakoshi T, Tabuse H and Shibata Y: Safety and efficacy of proton-beam radiation therapy for patients with locally recurrent rectal cancer. Annals of Oncology 23: xi160, 2020. DOI: $10.1016 / \mathrm{s} 0923-7534(20) 32506-0$

21 Berman A, Both S, Sharkoski T, Goldrath K, Tochner Z, Apisarnthanarax S, Metz J and Plastaras J: Proton reirradiation of recurrent rectal cancer: Dosimetric comparison, toxicities, and preliminary outcomes. International Journal of Particle Therapy 1(1): 2-13, 2017. DOI: 10.14338/ijpt.13-00002.1

22 Kanai T, Endo M, Minohara S, Miyahara N, Koyama-ito H, Tomura H, Matsufuji N, Futami Y, Fukumura A, Hiraoka T, Furusawa Y, Ando K, Suzuki M, Soga F and Kawachi K: Biophysical characteristics of HIMAC clinical irradiation system for heavy-ion radiation therapy. Int J Radiat Oncol Biol Phys 44(1): 201-210, 1999. PMID: 10219815. DOI: 10.1016/s03603016(98)00544-6

23 Yamada S, Kamada T, Ebner DK, Shinoto M, Terashima K, Isozaki Y, Yasuda S, Makishima H, Tsuji H, Tsujii H, Isozaki T, Endo S, Takahashi K, Sekimoto M, Saito N, Matsubara H and Working Group on Locally Recurrent Rectal Cancer: Carbon-ion radiation therapy for pelvic recurrence of rectal cancer. Int J Radiat Oncol Biol Phys 96(1): 93-101, 2016. PMID: 27375166. DOI: $10.1016 /$ j.ijrobp.2016.04.022

24 Shinoto M, Yamada S, Okamoto M, Shioyama Y, Ohno T, Nakano T, Nemoto K, Isozaki Y, Kawashiro S, Tsuji $\mathrm{H}$ and Kamada T: Carbon-ion radiotherapy for locally recurrent rectal cancer: Japan Carbon-ion Radiation Oncology Study Group (JCROS) Study 1404 Rectum. Radiother Oncol 132: 236-240, 2019. PMID: 30360998. DOI: 10.1016/j.radonc.2018.10.007
25 Gerweck LE and Kozin SV: Relative biological effectiveness of proton beams in clinical therapy. Radiother Oncol 50(2): 135142, 1999. PMID: 10368035. DOI: 10.1016/s0167-8140(98) 00092-9

26 Law MP, Ahier RG and Field SB: The effect of prior heat treatment on the thermal enhancement of radiation damage in the mouse ear. Br J Radiol 52(616): 315-321, 1979. PMID: 444823. DOI: $10.1259 / 0007-1285-52-616-315$

27 Song CW: Effect of local hyperthermia on blood flow and microenvironment: a review. Cancer Res 44(10 Suppl): 4721s4730s, 1984. PMID: 6467226.

28 Wust P, Hildebrandt B, Sreenivasa G, Rau B, Gellermann J, Riess H, Felix R and Schlag PM: Hyperthermia in combined treatment of cancer. Lancet Oncol 3(8): 487-497, 2002. PMID: 12147435. DOI: 10.1016/s1470-2045(02)00818-5

29 Datta NR, Puric E, Schneider R, Weber DC, Rogers S and Bodis $\mathrm{S}$ : Could hyperthermia with proton therapy mimic carbon ion therapy? Exploring a thermo-radiobiological rationale. Int J Hyperthermia 30(7): 524-530, 2014. PMID: 25314095. DOI: $10.3109 / 02656736.2014 .963703$

30 Kemmochi A, Hisakura K, Terashima H, Akashi Y, Sakurai H and Ohkohchi N: Surgical spacer insertion for proton beam therapy to the near-gastrointestinal tumor. Report of six cases. Nihon Rinsho Geka Gakkai Zasshi (Journal of Japan Surgical Association) 75(5): 1164-1168, 2021. DOI: 10.3919/jjsa.75.1164

31 Akasaka H, Sasaki R, Miyawaki D, Mukumoto N, Sulaiman NS, Nagata M, Yamada S, Murakami M, Demizu Y and Fukumoto T: Preclinical evaluation of bioabsorbable polyglycolic acid spacer for particle therapy. Int J Radiat Oncol Biol Phys 90(5): 1177-1185, 2014. PMID: 25539373. DOI: 10.1016/j.ijrobp. 2014.07.048
Received February 4, 2021

Revised May 24, 2021

Accepted May 26, 2021 